\title{
Expression of Adipocyte-Associated Genes in the Mouse Tail Epididymal Fat at Different Postnatal Ages
}

\author{
${ }^{\dagger} \mathrm{Ki}$-Ho Lee \\ Dept. of Biochemistry and Molecular Biology, College of Medicine, Eulji University, Daejeon 34824, Korea
}

Received: July 28, 2020

Revised: August 25, 2020

Accepted: September 1, 2020

${ }^{\dagger}$ Corresponding author

Ki-Ho Lee

Dept. of Biochemistry and Molecular

Biology, Eulji University, Daejeon 34824,

Korea.

Tel: +82-42-259-1643

Fax: +82-42-259-1649

E-mail: kiholee@eulji.ac.kr

Copyright $\odot 2020$ The Korean Society of Developmental Biology.

This is an Open Access article distributed under the terms of the Creative Commons Attribution Non-Commercial License (http://creativecommons.org/licenses/ by-nc/4.0/) which permits unrestricted non-commercial use, distribution, and reproduction in any medium, provided the original work is properly cited.

ORCID

Ki-Ho Lee

https://orcid.org/0000-0002-3495-5126

Conflict of interests

The authors declare no potential conflict of interest.

Acknowledgements

This research was supported by Basic Science Research Program through the National Research Foundation of Korea (NRF) funded by the Ministry of Education (2017R1D1A 1B03030209).

Authors' contributions

The article is prepared by a single author.

Ethics approval

All procedures were carried out in accordance with the guidelines for the care and use of laboratory animals of National Research Council in Korea.

\section{Abstract}

The fat pad defined as the epididymal fat is located at the head part of the epididymis. However, another fat mass is present near the caudal epididymis, named tail epididymal fat. The present research was focused to determine the expression of adipocyte-associated molecules in the mouse tail epididymal fat at different postnatal ages, including 2,5 , 8, and 12 months of age. The quantitative real-time PCR analysis showed continuous increases of expression levels of delta like non-canonical Notch ligand 1, leptin, and resistin as postnatally aged. The transcript level of peroxisome proliferator-activated receptor gamma was the highest at 5 months of age, remaining at a steady level until 12 months of age. Expression levels of fatty acid binding protein 4 , leptin, and adiponectin were significantly increased until 8 months of age, persisting the level at 12 months of age. The transcript level of fatty acid synthase was significantly increased at 8 months of age, without a further change of the level at 12 months of age. These findings exhibited the expression of adipocyte-associated genes which were also detected at the ordinary epididymal fat pad. However, expression patterns of these genes in the tail epididymal fat are different with those in the distal and proximal epididymal fat, suggesting distinct characteristics and/or functions of the tail epididymal fat.

Keywords: Tail epididymal fat, Mouse, Real-time polymerase chain reaction (PCR), Gene expression, Adipocyte

\section{INTRODUCTION}

It is well defined that the epididymal fat is located near the head of the epididymis (Pond, 1999; Berry et al., 2013) (Fig. 1). As a part of gonadal adipose tissue, the epididymal fat is divided into two parts, distal and proximal epididymal fat, based on histochemical characteristics (Tirard et al., 2007). For instance, the proximal epididymal fat has higher aldo-keto reductase $1 \mathrm{~B} 7$, cyclooxygenase 2 , and GATA-3 transcript levels than the distal epididymal fat (Tirard et al., 2007). In addition, our recent researches have shown different expression patterns of some adipocyte-associated molecules during postnatal period (Lee, 2019; Lee \& Kim, 2019). Expression of leptin (Lep) in the distal epididymal fat is continuously increased as aged (Lee \& Kim, 2019), while the highest level of Lep in the proximal epididymal fat is detected at 8 months of age, followed by a significant drop at 12 months of age (Lee, 2019). Similar findings are found in the expression patterns of fatty acid synthase (Fasn) and lipoprotein lipase (Lpl), resistin (Retn), adiponectin (Adipoq), and fatty acid binding protein 4 (Fabp4) (Lee, 2019; 


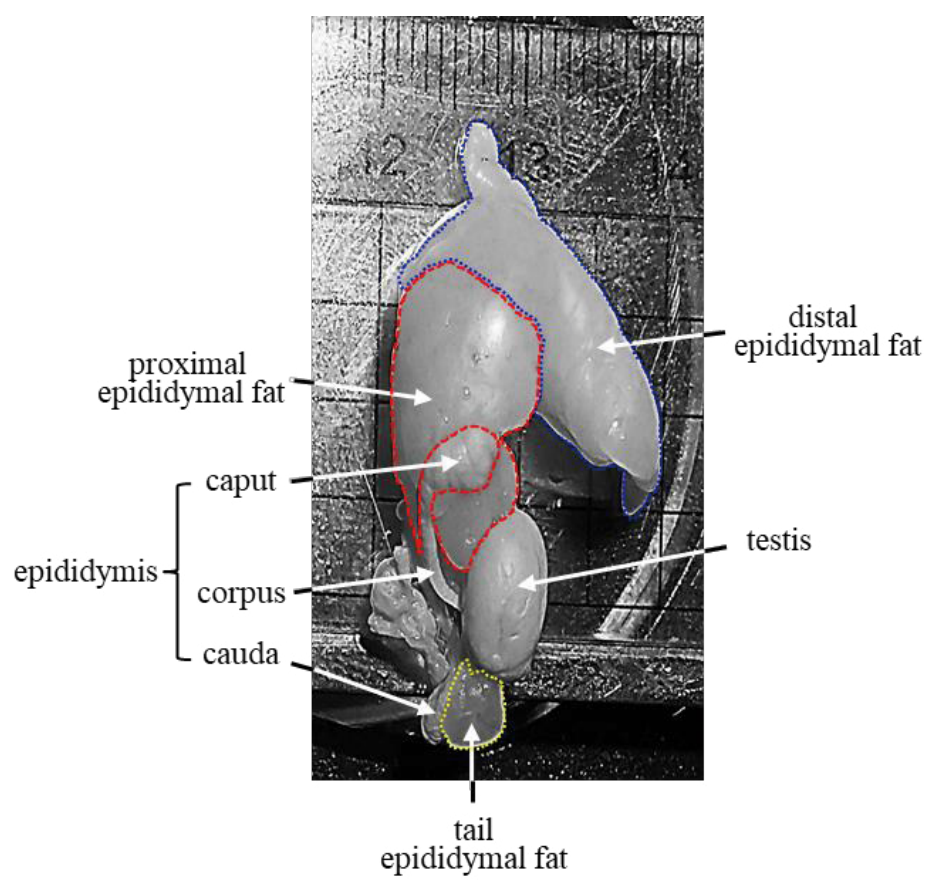

Fig. 1. A representative photograph of the adult mouse male reproductive tract. The ordinary epididymal fat pad is consisted of distal and proximal epididymal parts and is located between the testis and the caput epididymis. Note that the tail epididymal fat is observed near the caudal epididymis and is relatively smaller size than the ordinary epididymal fat, distal and proximal epididymal fat.

Lee \& Kim, 2019). Differential expression patterns of adipocyte-associated genes in two epididymal fat regions suggest that these two regions of epididymal fat would have different developmental pathways and thereby biological functions.

The epididymal fat is derived from non-adipogenic multipotent mesenchymal progenitor cells at the very early postnatal age (Han et al., 2011). The differentiation and development into the pre- and mature epididymal adipocyte of the progenitor cells are dependent upon the cell-matrix and cell-cell contact and appear to begin as early as 4 days of postnatal age in the mouse (Han et al., 2011). The mature adipocyte-like cells are first observed around 14 days of postnatal age, and the distal epididymal fat likely contributes to proper development of the epididymal fat at the adult (Han et al., 2011). The development of epididymal fat during postnatal period is associated with enlargement of size and increase of adipocyte number (Cleary et al., 1977). The growth of epididymal fat at the early stage of postnatal development is mostly related with proliferation of adipocyte, while the size expansion of each adipocyte particularly influences on the development of epididymal fat at late postnatal stage (Cleary et al., 1977; Gruen et al., 1980). Even though the detailed molecular mechanism of epididymal fat growth during postnatal period has not been addressed, it is believed that the general pathway involving in sequential actions of various adipogenic genes required for proliferation and maturation of adipocyte would be applicable to postnatal development of the epididymal fat (Hausman et al., 2001).

Other than the ordinary epididymal fat placed between the testis and the epididymis, an additional fat deposit, named the tail epididymal fat, exists near the caudal epididymis (Fig. 1). The tail epididymal fat is much smaller than the ordinary epididymal fat and appears to begin around 10 days of postnatal age (personal observation). Like the ordinary epididymal fat, the tail epididymal fat continuously grows in size as aged (personal observation). However, it is hard to find detailed description of the tail epididymal fat, and no careful examination of the tail epididymal fat has not 
been conducted yet. In spite of a lack of scientific information about the tail epididymal fat, the being at anatomically different place from the ordinary epididymal fat has been held an attention for the biological examination.

Our previous researches have shown the existence and different expression patterns of some adipocyte-associated molecules in the distal and proximal epididymal fat in mouse and whole epididymal fat in rat at various postnatal ages (Lee \& Kim, 2018; Lee, 2019; Lee \& Kim, 2019). Thus, the present research was focused to determine whether expression of adipocyte-associated molecules found in the ordinary epididymal fat could be detected in the tail epididymal fat using quantitative real-time polymerase chain reaction (PCR). In addition, expression patterns of these molecules in the tail epididymal fat during postnatal period was compared with those in the ordinary epididymal fat.

\section{MATERIALS AND METHODS}

\section{Experimental animals and collection of the tail epididymal fat}

A total of 24 male C57BL/6N mice at 1 month of postnatal age were purchased from Samtako (Osan, Korea), and randomly allocated into 4 different age groups, 2, 5, 8, and 12 months of age, at the arrival. Each age group had six mice, and experimental animals were allowed for food and water ad libitum under controlled environment (14-h/10-h light/dark cycle) for a whole experimental period. All procedures were carried out in accordance with the guidelines for the care and use of laboratory animals of National Research Council in Korea.

When the animal reached a proper postnatal age, the animal was euthanized by $\mathrm{CO}_{2}$ stunning in a closed plastic chamber. The male reproductive tract, including testis, epididymis, and epididymal fat pad, was pulled out throughout a lower abdominal incision and was placed in cold PBS. The epididymis was separated from the testis, and the tail epididymal fat near the caudal epididymis was carefully isolated from the rest of epididymal part and vas deferens. The collected tail epididymal fat was washed once in fresh cold PBS and rapidly placed in liquid nitrogen and then $-80^{\circ} \mathrm{C}$ freezer. Because the tail epididymal fat collected from an experimental animal is not sufficient to obtain total RNA aliquot for quantitative real-time PCR analysis, all tail epididymal fat tissues in an experimental age group were pooled to acquire adequate amount of total RNA extract.

\section{Extraction of total RNA from the tissue sample and analysis of quantitative real-time polymerase chain reaction (PCR)}

A pool of the tail epididymal fat was homogenized in $1 \mathrm{~mL}$ of TRI REAGENT solution (Molecular Research Center, Cincinnati, OH, USA). The total RNA pellet was obtained from sequential addition of chloroform and isopropanol into tissue homogenate and resuspended in DEPC-treated $\mathrm{dH}_{2} \mathrm{O}$. The quantity and quality of total RNA extract were determined by a spectrophotometry (NanoDrop Lite, Thermo Scientific, Wilmington, DE, USA) and 1.2\% agarose gel electrophoresis, respectively.

To generate the first strand of complementary DNA (cDNA) for quantitative real-time PCR analysis, $1 \mu \mathrm{g}$ of total RNA was mixed with pre-made reverse transcription (RT) mixture (iScrip ${ }^{\mathrm{TM}}$ Reverse transcription Supermix for RT-qPCR, Bio-Rad Laboratories, Hercules, CA, USA) and nuclease free- $\mathrm{dH}_{2} \mathrm{O}$ to make the final volume of $20 \mu \mathrm{L}$. The $\mathrm{RT}$ reaction was carried out in $25^{\circ} \mathrm{C}$ for $5 \mathrm{~min}, 46^{\circ} \mathrm{C}$ for $20 \mathrm{~min}$, and $95^{\circ} \mathrm{C}$ for $1 \mathrm{~min}$.

The constructed cDNA was directly utilized for quantitative real-time PCR analysis. The same PCR primers employed from previous researches for mouse distal and proximal epididymal fat 
tissue (Lee, 2019; Lee \& Kim, 2019) were used for the current study. The detailed information for oligonucleotide primers is listed in Table 1 . The PCR mixture was prepared with $1 \mu \mathrm{L}$ of cDNA, 10 pmol of each primer, $7 \mu \mathrm{L}$ of $\mathrm{iQ}^{\mathrm{TM}} \mathrm{SYBR}^{\circledR}$ Green Supermix (Bio-Rad Laboratories), and nuclease free- $\mathrm{dH}_{2} \mathrm{O}$ to adjust the final reaction volume. The PCR condition is as follows; a pre-denaturation at $95^{\circ} \mathrm{C}$ for $5 \mathrm{~min}$, cycles of a denaturation at $95^{\circ} \mathrm{C}$ for $30 \mathrm{sec}$, an annealing at $\mathrm{T}_{\mathrm{m}}$ for $30 \mathrm{sec}$, and an extension at $72^{\circ} \mathrm{C}$ for $30 \mathrm{sec}$, and an additional extension at $72^{\circ} \mathrm{C}$ for $10 \mathrm{~min}$. The presumed size of PCR product was checked with $1.2 \%$ agarose gel electrophoresis.

\section{Statistical analysis and presentation of data}

Independently quadruped RT reactions and PCRs were performed to obtain a mean and standard error for the expression level of each target molecule at a given postnatal age. The expression level of a target gene was normalized to that of $18 \mathrm{~S}$ ribosomal RNA (Rn18s), an internal control of PCR analysis and was presented in the relative ratio against that of $R n 18$ s in a graph.

The existence of statistical difference among the transcript levels of a target gene at different postnatal ages was first evaluated by one-way ANOVA. If significance present, a post-hoc analysis, Duncan's test, was followed to determine statistical difference among age groups. If $p$-value was less than 0.05 , it was judged as statistically significant on expression level of the target gene between those different ages and then denoted by different letters in the result graph.

\section{RESULTS}

\section{Postnatal expression of Pparg and Fabp4 in the mouse tail epididymal fat tissue}

Expression patterns of Pparg and Fabp4 are shown in Fig. 2. The level of Pparg transcript was significantly increased at 5 months of age, compared with its at 2 months of age (Fig. 2A). However, expression level of Pparg gene was not changed at 8 months of age and even at 12 months of age (Fig.

Table 1. Primers for real-time PCR analysis

\begin{tabular}{|c|c|c|c|}
\hline Gene & $\begin{array}{l}\text { Primer sequence } \\
\qquad\left(5^{\prime} \rightarrow 3^{\prime}\right)\end{array}$ & $\begin{array}{c}\mathrm{T}_{\mathrm{m}} \\
\left({ }^{\circ} \mathrm{C}\right)\end{array}$ & $\begin{array}{l}\text { Expected size } \\
\text { (bps) }\end{array}$ \\
\hline $\begin{array}{l}\text { Pparg } \\
\text { (U01841.1) }\end{array}$ & $\begin{array}{l}\text { (F) GATTCATGACCAGGGAGTTC } \\
\text { (R) GACTCTGGGTGATTCAGCTT }\end{array}$ & 60 & 247 \\
\hline $\begin{array}{l}\text { Fabp4 } \\
\text { (NM_024406.3) }\end{array}$ & $\begin{array}{l}\text { (F) CAAGCCCAACATGATCATCAGC } \\
\text { (R) CACGCCCAGTTTGAAGGAAATC }\end{array}$ & 60 & 97 \\
\hline $\begin{array}{l}\text { Dlk1 } \\
\text { (NM_010052.5) }\end{array}$ & $\begin{array}{l}\text { (F) GGCCATCGTCTTTCTCAACA } \\
\text { (R) CTCCTCATCACCAGCCTCCT }\end{array}$ & 60 & 181 \\
\hline $\begin{array}{l}\text { Fasn } \\
\text { (NM_011480.3) }\end{array}$ & $\begin{array}{l}\text { (F) GTGTGGACATGGTCACAGATG } \\
\text { (R) GACCGCTTGGGTAATCCATA }\end{array}$ & 60 & 67 \\
\hline $\begin{array}{l}\text { Lpl } \\
\text { (NM_008509.2) }\end{array}$ & $\begin{array}{l}\text { (F) TCAGAGCCAAGAGAAGCAGCA } \\
\text { (R) TTGTGTTGCTTGCCATCCTCA }\end{array}$ & 60 & 118 \\
\hline $\begin{array}{l}\text { Lep } \\
\text { (NM_008493.3) }\end{array}$ & $\begin{array}{l}\text { (F) TTCACACACGCAGTCGGTAT } \\
\text { (R) TCATTGGCTATCTGCAGCAC }\end{array}$ & 55 & 165 \\
\hline $\begin{array}{l}\text { Adipoq } \\
\text { (NM_009605.5) }\end{array}$ & $\begin{array}{l}\text { (F) AGGCCGTTCTCTTCACCTACG } \\
\text { (R) GACTTGGTCTCCCACCTCCAGA }\end{array}$ & 62 & 95 \\
\hline $\begin{array}{l}\text { Retn } \\
\text { (NM_022984.3) }\end{array}$ & $\begin{array}{l}\text { (F) TTCCTTGTCCCTGAACTGCT } \\
\text { (R) TCAAGACTGCTGTGCCTTCT }\end{array}$ & 55 & 187 \\
\hline $\begin{array}{l}\text { Rn18s } \\
\text { (NR_03278.3) }\end{array}$ & $\begin{array}{l}\text { (F) ACTCAACACGGGAAACCTCA } \\
\text { (R) AACCAGACAAATCGCTCCAC }\end{array}$ & 55 & 123 \\
\hline
\end{tabular}

PCR, polymerase chain reaction; Pparg, peroxisome proliferator-activated receptor, gamma; Fabp4, fatty acid binding protein 4; Dlk1, delta like non-canonical Notch ligand 1; Fasn, fatty acid synthase; Lpl, lipoprotein lipase; Lep, leptin; Adipoq, adiponectin; Retn, resistin; Rn18s, 18S ribosomal RNA. 
(A)

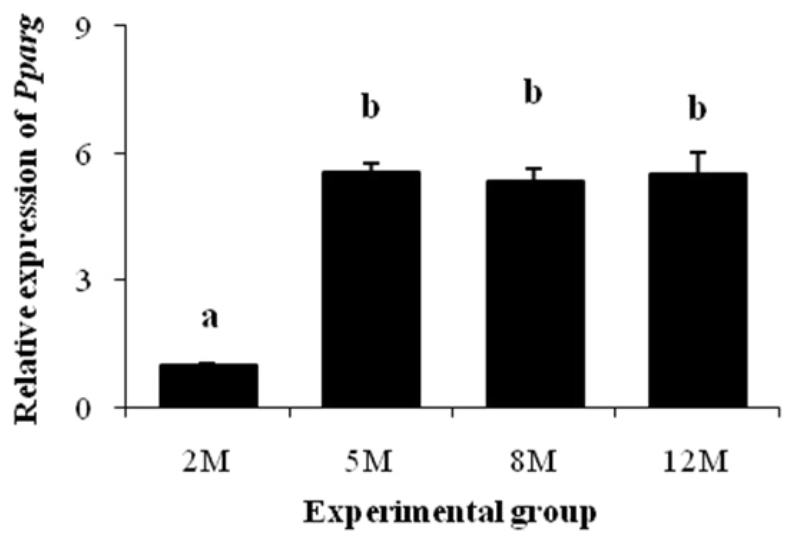

(B)

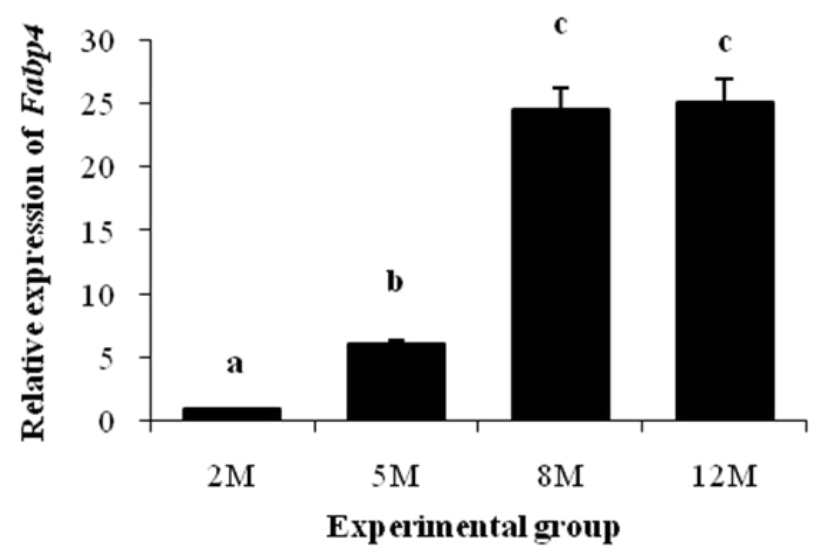

Fig. 2. Postnatal expression of Pparg and Fabp4 genes in the mouse tail epididymal fat. The relative expression ratios of $P$ parg (A) and Fabp4 (B) transcripts are shown. Different letters indicate statistical significances at $p<0.05 . \mathrm{M}$, months of postnatal age; Pparg, peroxisome proliferator-activated receptor, gamma; Fabp4, fatty acid binding protein 4.

2A).

The transcript level of Fabp4 at 5 months of age was also significantly higher than its at 2 months of age (Fig. 2B). However, unlike Pparg, an additional huge increase of Fabp4 mRNA level was detected at 8 months of age (Fig. 2B). The expression level of Fabp4 at 12 months of age was not different with its at 8 months of age (Fig. 2B).

\section{Postnatal expression of DIk1 and Fasn in the mouse tail epididymal fat tissue}

Expression level of Dlk1 gene at 5 months of age was significantly increased than its at 2 months of age (Fig. 3A). The transcript level of Dlk1 at 8 months of age was about 3-fold higher than its at 5 months of age (Fig. 3A). An additional over 2-times increase of Dlk1 transcript level was detected at 12 months of age (Fig. 3A).

There was no significant change of Fasn transcript level at 5 months of age, compared with its at 2 months of age (Fig. 3B). However, the level of Fasn mRNA was significantly increased at 8 months of age, followed by a steady level at 12 months of age (Fig. 3B).

\section{Postnatal expression of $L p /$ and $L e p$ in the mouse tail epididymal fat tissue}

Expression of $L p l$ gene was significantly increased at 5 months of age, compared with the level of $L p l$ transcript at 2 months of age (Fig. 4A). A further increase of $L p l$ transcript level was observed at 8 months of age (Fig. 4A). However, there was no significant expression change of $L p l$ between 8 and 12 months of age (Fig. 4A).

As shown in Fig. 4B, a significant increase of Lep transcript level at 5 months of age was detected, compared with its at 2 months of age. An additional increase of Lep mRNA level was found at 8 months of age, followed by a further rise of $L e p$ transcript level at 12 months of age (Fig. 4B).

\section{Postnatal expression of Adipoq and Retn in the mouse tail epididymal fat tissue}

About 5 -fold increase of Adipoq transcript level was detected at 5 months of age, compared with at 2 months of age (Fig. 5A). A further significant increase of Adipog gene expression was observed at 8 months of age (Fig. 5A). But, the Adipoq transcript level at 12 months of age was not 
(A)

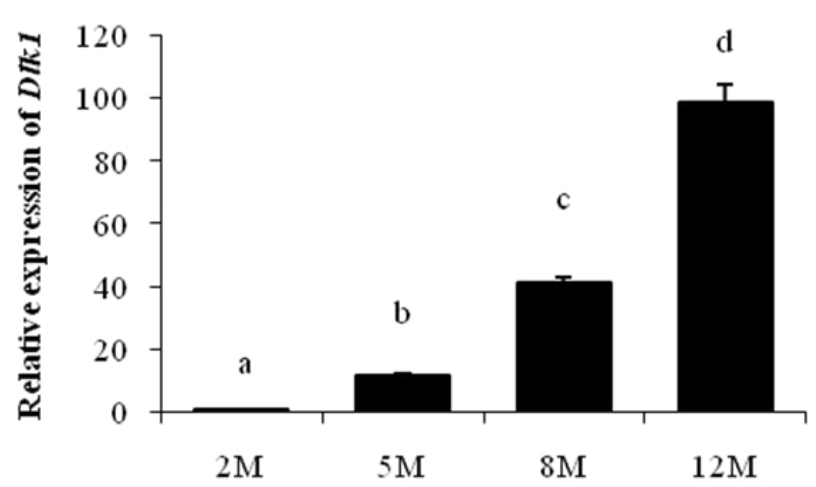

Experimental group
(B)

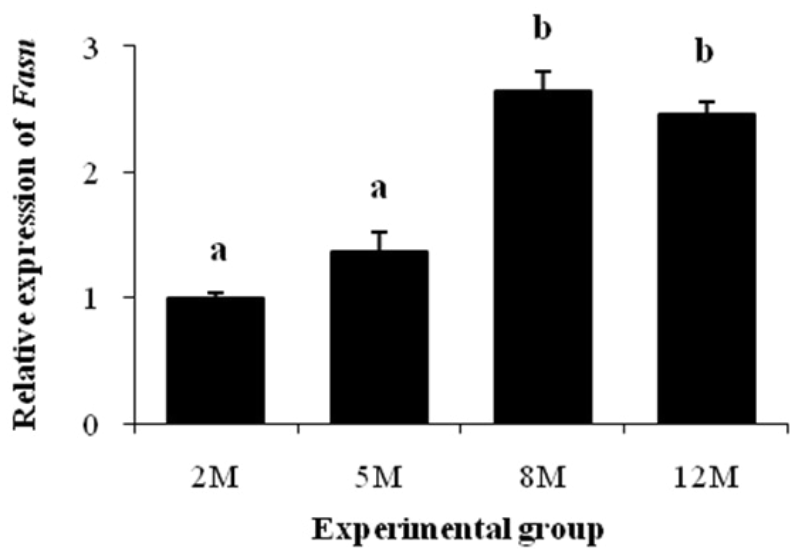

Fig. 3. Postnatal expression of Dlk1 and Fasn genes in the mouse tail epididymal fat. The relative expression ratios of Dlk1 (A) and Fasn (B) transcripts are shown. Different letters indicate statistical significances at $p<0.05 . \mathrm{M}$, months of postnatal age; Dlk1, delta like non-canonical Notch ligand 1; Fasn, fatty acid synthase.

(A)

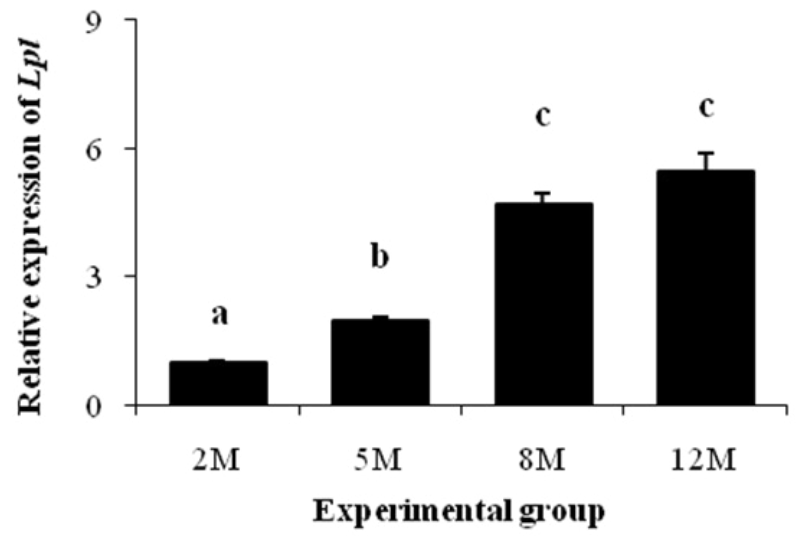

(B)

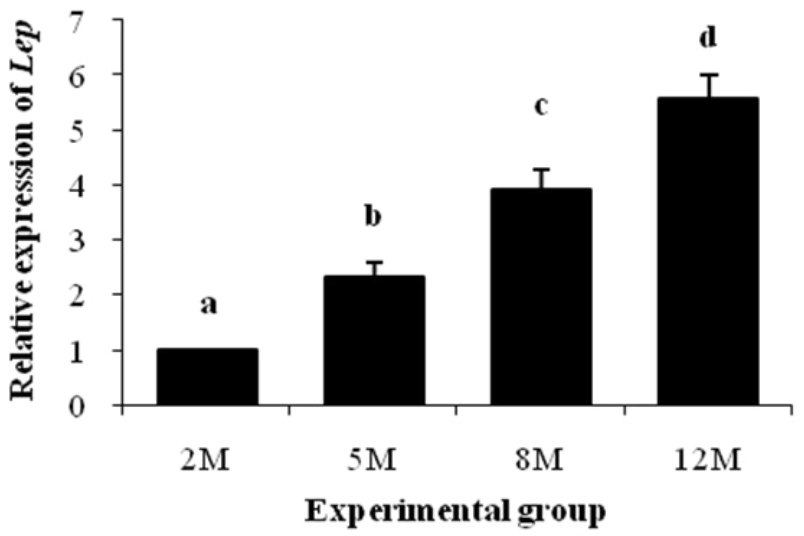

Fig. 4. Postnatal expression of $L p l$ and $L e p$ genes in the mouse tail epididymal fat. The relative expression ratios of $L p l(\mathrm{~A})$ and $L e p(\mathrm{~B})$ transcripts are shown. Different letters indicate statistical significances at $p<0.05$. M, months of postnatal age; $L p l$, lipoprotein lipase; Lep, leptin.

statistically different with its at 8 months of age (Fig. 5A).

The transcript level of Retn at 5 months of age was significantly higher than its at 2 months of age (Fig. 5B). Then, an additional increase of Retn transcript level was detected at 8 months of age (Fig. 5B). The highest expression level of Retn gene among experimental age groups was found at 12 months of age (Fig. 5B).

\section{DISCUSSION}

The presence and nature of the tail epididymal fat have not been recognized, presumably due 
(A)

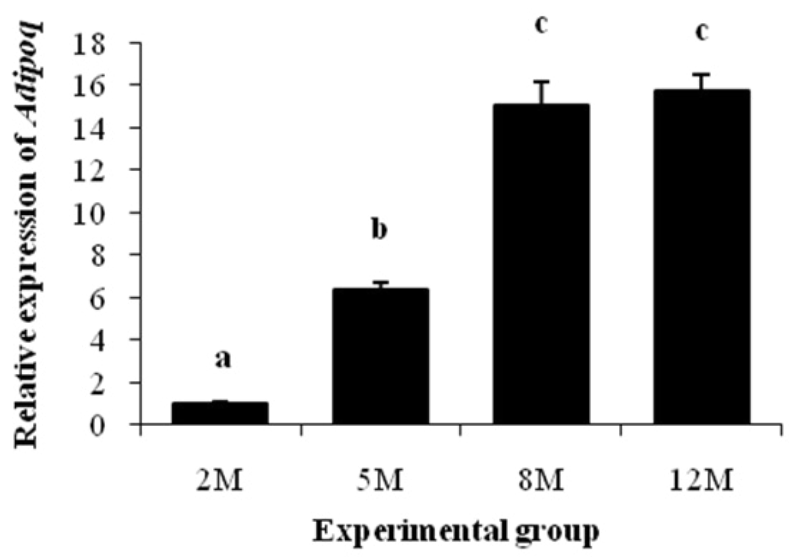

(B)

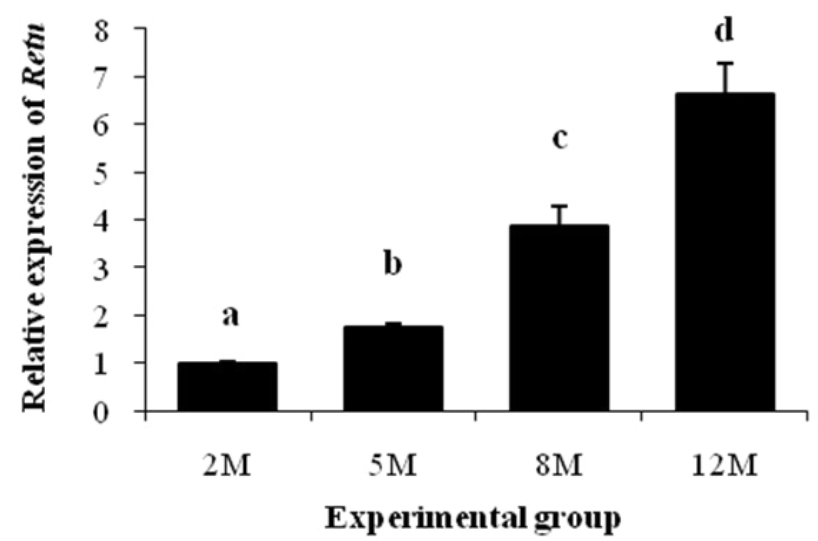

Fig. 5. Postnatal expression of Adipoq and Retn genes in the mouse tail epididymal fat. The relative expression ratios of $A d i p o q$ (A) and Retn (B) transcripts are shown. Different letters indicate statistical significances at $p<0.05$. M, months of postnatal age; Adipoq, adiponectin; Retn, resistin.

to small size and the location isolated from the ordinary epididymal fat. In the present research, the presence and expression patterns of adipocyte-associated molecules found in the ordinary epididymal fat at different postnatal ages have been investigated in the tail epididymal fat by quantitative real-time PCR analysis. The research data show that the transcripts of adipocyteassociated genes examined are indeed present in the tail epididymal fat. In addition, expression levels of these genes during postnatal period are mostly higher at the elderly age than those at the younger age.

The differentiation of preadipocyte into mature adipocyte and maintenance of differentiated status of adipocyte require a proper expression of Pparg (Tamori et al., 2002). Interestingly, the expression patterns of Pparg in the distal and proximal epididymal fat during postnatal period are slightly different (Lee, 2019; Lee \& Kim, 2019). That is, the transcript level of Pparg in the distal epididymal fat is continuously increased as aged (Lee \& Kim, 2019), while Pparg transcript level in the proximal epididymal fat is significant decreased at 5 months of age, followed by the highest level of Pparg at 8 months of age (Lee, 2019). In the current findings, the level of Pparg expression in the tail epididymal fat is significantly increased at 5 months of age and remained at constant level until 12 months of age. These results imply that the maturation of each epididymal fat segment by the action of Pparg is likely differentially regulated during postnatal period. From in vitro experiment with adipocytes derived from different epididymal fat parts, the modification of Pparg expression would provide information to assess a role of Pparg on the postnatal maturation of the epididymal fat.

The expression of Fabp4 in various adipocyte tissue has been revealed in previous researches (Kletzien et al., 1992). As a promoting factor for lipid storage in adipocyte, it is reasonable to consider that expression of Fabp4 would be increased as fat tissue becomes larger with aging. Indeed, the present showed that Fabp4 transcript levels at 8 and 12 months of age were over 20-folds higher than that at 2 months of age, indicating the occurrence of considerable accumulation of lipid droplet within the tail epididymal fat cells at the later postnatal age. Expression patterns of Fabp4 in the distal and proximal epididymal fat during postnatal period are not same with that in the tail epididymal fat. In the proximal epididymal fat, a significant increase of Fabp4 expression is observed at 8 months of age (Lee, 2019). However, Fabp4 transcript level in the distal epididymal 
fat is continuously increased as ages (Lee \& Kim, 2019). The present study shows that expression pattern of Fabp4 in the tail epididymal fat until 8 months of postnatal age is quite similar with that in the distal epididymal fat, even though an additional significant increase of Fabp4 expression at 12 months of age is detected in the distal epididymal fat (Lee, 2019). From these observations, it is presumed that lipid accumulation in the distal, proximal, and tail epididymal fats actively occurs around 8 months of age.

The Dlk1 promotes an inhibition of adipogenesis and suppress the differentiation of preadipocyte into adipocyte, resulting in higher expression of $D l k 1$ in preadipocyte than mature adipocyte (Wang \& Sul, 2009). In the distal and proximal epididymal fat, the extraordinary increases of Dlk1 transcript level are observed at 8 months of age (Lee, 2019; Lee \& Kim, 2019). Even though the magnitude of surge in Dlk expression is slightly different, the Dlk transcript levels in the tail epididymal fat are also increased as aged. Together, these findings imply that Dlk plays a role on the maturation of the epididymal fat during postnatal period, other than an inhibitory effect on adipogenesis, because the differentiation of preadipocyte in the epididymal fat seems to be completed before 1 month of postnatal age (Han et al., 2011). An examination of functional role of Dlk in the differentiated adipocyte would be helpful to understand how Dlk influences on the maturation of the epididymal fat during postnatal period.

The LPL promotes lipolysis and FASN stimulates lipogenesis in adipocyte (Proença et al., 2014). Thus, the balanced actions of LPL and FASN are necessary to regulate the storage of fatty acid in adipocyte. Because the cell size and lipid storage of the epididymal adipocyte at the elderly age is larger than those at the younger age (Björntorp et al., 1979), it is considerable that expression of $L p l$ would be decreased and the level of Fasn transcript would be increased as aged. However, the present research has shown significantly high levels of both $L p l$ and Fasn expression at the elderly age. Similar expression patterns of $L p l$ and Fasn at the tail epididymal fat are observed at the distal and proximal epididymal fat during postnatal age (Lee, 2019; Lee \& Kim, 2019). It is not possible to provide a clear explanation about the increase of $L p l$ expression in the epididymal fat with aging at this point. A possible suggestion would be that the increase of $L p l$ transcript level does not always reflect the increase of functional LPL protein level, due to different transcription and translation efficiencies of gene expression. An additional examination at protein level could deliver clear information to explain such expression pattern of $L p l$ in the epididymal fat during postnatal period.

The crosstalk between adipocyte and metabolic tissues for regulation of systemic energy expenditure is usually controlled by adipocytokines secreted from the adipose tissue, such as leptin, adiponectin, and resistin (Haiming, 2014). The biological functions of these adipocytokines include the control of food intake and energy usage by leptin, reduced insulin resistance and thus increased insulin action by adiponectin, and an increase of insulin resistance by resistin (Haiming, 2014). The function of adiponectin in the male reproductive tract has not been clearly defined, in spite of the presence of adiponectin receptor type 1 and 2 in the testis and epididymis (Singh et al., 2018). A possible positive regulatory effect of resistin on steroidogenic activity in the testicular Leydig cell has been suggested (Roumaud \& Martin, 2015). Even though the exact role of leptin on the male reproductive function has not been revealed, it is proposed that leptin would involve in suppression of testicular steroidogenesis (Zhang \& Gong, 2018). These findings reflex the existence of a complex regulatory mechanism on the male reproduction by adipocytokines. Chu et al. (2010) have demonstrated that the presence of ordinary epididymal fat is essential for maintaining spermatogenesis, suggesting possible role of adipokines originated from the ordinary epididymal fat on the regulation of spermatogenesis in the testis. Thus, based on the findings of Lep, Adipoq, and Retn expression in the tail epididymal fat from the present study, it is reasonable to consider that the tail epididymal fat could have an effect on the testicular function. 
The current findings have the significances on the examination of the presence and expression patterns of various adipocyte-associate genes in the mouse tail epididymal fat at different postnatal ages, presumably for the first time. Expression patterns of these genes distinguished from those in the ordinary epididymal fat imply the existence of distinct role of the tail epididymal fat. Even though the data from present research does not give a direct information of an importance of the tail epididymal fat, further examination of this tissue would provide an additional evidence of a functional role of epididymal fat on the male reproduction, such as regulation of testicular and/or epididymal function.

\section{REFERENCES}

Berry DC, Stenesen D, Zeve D, Graff JM (2013) The developmental origins of adipose tissue. Development 140:3939-3949.

Björntorp P, Karlsson M, Gustafsson L, Smith U, Sjöström L, Cigolini M, Storck G, Pettersson P (1979) Quantitation of different cells in the epididymal fat pad of the rat. J Lipid Res 20:97106.

Chu Y, Huddleston GG, Clancy AN, Harris RBS, Bartness TJ (2010) Epididymal fat is necessary for spermatogenesis, but not testosterone production or copulatory behavior. Endocrinology 151:5669-5679.

Cleary MP, Greenwood MRC, Brasel JA (1977) A multi-factor analysis of growth in the rat epididymal fat pad.J Nutr 107:1969-1974.

Gruen R, Kava R, Greenwood MR (1980) Development of basal lipolysis and fat cell size in the epididymal fat pad of normal rats. Metabolism 29:246-253.

Haiming C (2014) Adipocytokines in obesity and metabolic disease.J Endocrinol 220:T47-T59.

Han J, Lee JE, Jin J, Lim JS, Oh N, Kim K, Chang SI, Shibuya M, Kim H, Koh GY (2011) The spatiotemporal development of adipose tissue. Development 138:5027-5037.

Hausman DB, DiGirolamo M, Bartness TJ, Hausman GJ, Martin RJ (2001) The biology of white adipocyte proliferation. Obes Rev 2:239-254.

Kletzien RF, Foellmi LA, Harris PK, Wyse BM, Clarke SD (1992) Adipocyte fatty acid-binding protein: Regulation of gene expression in vivo and in vitro by an insulin-sensitizing agent. Mol Pharmacol 42:558-562.

Lee KH (2019) Postnatal expressional patterns of adipose-associated molecules in the mouse proximal epididymal fat. Dev Reprod 23:313-322.

Lee KH, Kim NH (2018) Expressional patterns of adipocyte-associated molecules in the rat epididymal fat during postnatal development period. Dev Reprod 22:351-360.

Lee KH, Kim NH (2019) Differential expression of adipocyte-related molecules in the distal epididymal fat of mouse during postnatal period. Dev Reprod 23:213-221.

Pond CM (1999) Physiological specialisation of adipose tissue. Prog Lipid Res 38:225-248.

Proença ARG, Sertié RAL, Oliveira AC, Campaña AB, Caminhotto RO, Chimin P, Lima FB (2014) New concepts in white adipose tissue physiology. Braz J Med Biol Res 47:192-205.

Roumaud P, Martin LJ (2015) Roles of leptin, adiponectin and resistin in the transcriptional regulation of steroidogenic genes contributing to decreased Leydig cells function in obesity. Horm Mol Biol Clin Investig 24:25-45.

Singh A, Choubey M, Bora P, Krishna A (2018) Adiponectin and chemerin: Contrary adipokines in regulating reproduction and metabolic disorders. Reprod Sci 25:1462-1473.

Tamori Y, Masugi J, Nishino N, Kasuga M (2002) Role of peroxisome proliferator-activated 
receptor- $\gamma$ in maintenance of the characteristics of mature 3T3-L1 adipocytes. Diabetes 51:2045-2055.

Tirard J, Gout J, Lefrançois-Martinez AM, Martinez A, Begeot M, Naville D (2007) A novel inhibitory protein in adipose tissue, the aldo-keto reductase AKR1B7: Its role in adipogenesis. Endocrinology 148:1996-2005.

Wang Y, Sul HS (2009) Pref-1 regulates mesenchymal cell commitment and differentiation through Sox9. Cell Metab 9:287-302.

Zhang J, Gong M (2018) Review of the role of leptin in the regulation of male reproductive function. Andrologia 50:e12965. 